\title{
Perpetrare è cosa da non perpetuare
}

\author{
Edoardo Lombardi Vallauri
}

PUBBLICATO: 02 OCTOBER 2020

\section{Quesito:}

Ci sono giunte diverse domande sul verbo perpetrare. Un primo gruppo di lettori chiede un parere su usi di questo verbo in contesti dove sembrerebbero più opportuni verbi diversi, come perpetuare, o addirittura punire. Altri chiedono quale ne sia il senso in contesti dove risulta di non facile comprensione, e se esistano dei sinonimi con cui sostituirlo.

\section{Perpetrare è cosa da non perpetuare}

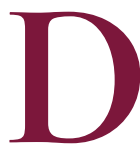

al Belgio, un lettore segnala che in un articolo pubblicato sul "Corriere della Sera" del i6 febbraio 2014 ha trovato questa frase: "Una città abituata a perpetrare le sue gerarchie si riconosce nel giovanotto venuto dal contado". Abbiamo controllato l'articolo, di Aldo Cazzullo, che riguarda la personalità e l'ascesa politica di Matteo Renzi. La città in questione è dunque Firenze, e il giovanotto venuto dal contado è il promettente Matteo, che proviene da Rignano sull'Arno. Il contesto permette di capire che il valore della frase è concessivo: benché abituata a non cambiare facilmente guida, Firenze questa volta sceglie come suo sindaco Renzi (preferendolo a personaggi più "storici", come il dirigente PD e già parlamentare europeo Lapo Pistelli, o l'ex portiere della fiorentina Giovanni Galli). Insomma, nel testo vi è un errore: perpetrare sta semplicemente al posto di perpetuare, che significa 'rendere perpetuo', 'far durare (a lungo, al limite in eterno)'.

Se si tratti di mero refuso o di vero scambio fra i due verbi, avvenuto in qualche fase della pubblicazione del testo, non è dato sapere con certezza; ma si possono fare delle ipotesi. Non per caso un'altra lettrice, da Torino, lamenta una certa frequenza di questa sostituzione, che esemplifica con l'espressione "perpetrare il ricordo di un evento", e ce ne chiede la causa. Un altro esempio ci viene segnalato da Milano, chiedendo se sia corretta questa frase, che la lettrice ha trovato leggendo un articolo su "Vanity Fair": "[...] aveva perpetrato il sogno di una famiglia". Non conoscendone il contesto, si può essere in dubbio. Ma facciamo un passo indietro per inquadrare il problema.

Perpetrare significa 'eseguire, commettere', e al tempo stesso presuppone che si tratti di azione illecita, ingiusta, colpevole. Non è l'unico verbo italiano a funzionare in questa maniera, e del resto il fenomeno si riscontra anche in altre lingue, come ad esempio l'inglese, su cui lo ha studiato per primo il linguista statunitense Charles Fillmore (I97I). Trasferendo l'analisi di Fillmore all'italiano, possiamo osservare che verbi di giudizio che sentiamo diversi come accusare e biasimare trasmettono però lo stesso insieme di informazioni. Consideriamo questi enunciati, in tempo di isolamento da coronavirus:

(I) Il carabiniere accusa Luigi di essere andato al parco.

(2) Il carabiniere biasima Luigi per essere andato al parco.

Entrambi gli enunciati si possono analizzare così:

Contenuto a. Il carabiniere dice qualcosa di Luigi 
Contenuto b. Luigi è andato al parco

Contenuto c. Andare al parco è male

La differenza fra (I) e (2) sta nella diversa presentazione delle informazioni (b) e (c). In (I) è asserito che il carabiniere attribuisca a Luigi l'azione di andare al parco, ed è dato per scontato che si tratti di azione sbagliata. Tecnicamente, si dice che questo contenuto è presupposto. Proprio per via della parte presupposta, è più normale dire "lo accusano di avere ucciso il vicino di casa" rispetto a "lo accusano di avere donato un milione per la costruzione di un ospedale": in ambo i casi si attribuisce a qualcuno una certa condotta, ma il verbo, presupponendo che si tratti di condotta riprovevole, è più adeguato all'attribuzione di omicidio che all'attribuzione di filantropia.

Possiamo dunque rappresentare (I) cosi:

\section{(I) Il carabiniere accusa Luigi di essere andato al parco.}

Asserito: Il carabiniere dice che Luigi è andato al parco

Presupposto: Andare al parco è male

All'inverso, biasimare asserisce che qualcosa sia male, presupponendo che sia stato fatto. Per questo è più normale dire "lo biasimano per avere guidato in modo imprudente" che "lo biasimano per essersi bevuto il lago di Iseo": in ambo i casi si giudica negativa una certa condotta, ma il verbo, presupponendo che quella condotta sia stata eseguita, è più adeguato all'attribuzione di guida spericolata che all'attribuzione di prosciugamento a sorsi di un grosso lago. La rappresentazione di (2) sarà la seguente:

\section{(2) Il carabiniere biasima Luigi per essere andato al parco.}

Asserito: Il carabiniere dice che andare al parco è male

Presupposto: Luigi è andato al parco

Dunque la differenza fra (I) e (2) è che il carabiniere nel primo dà per scontato che andare al parco è male e asserisce che Luigi l'ha fatto, mentre nel secondo dà per scontato che Luigi sia andato al parco e asserisce che questo è male.

Altri verbi di giudizio italiani che hanno contenuti simili ma distribuiscono diversamente che cosa è asserito e che cosa è presupposto sono criticare, rimproverare, giustificare, scusare, chiedere scusa, perdonare, lodare. Presupposizioni analoghe sono contenute nel significato di sperare, augurarsi, minacciare, temere ecc. Ognuno dei lettori può cimentarsi in analisi simili a quelle di cui abbiamo dato un esempio.

In ogni caso, ora sarà più chiaro come funziona il nostro perpetrare: asserisce che l'azione sia compiuta, e dà per scontato che sia riprovevole. Questo permette di rispondere anche al lettore che da Busto Arsizio ci chiede di "suggerire dei sinonimi adatti a sostituire il termine perpetrare, mantenendo le stesse sfumature di significato, nella frase Quando usi questo tipo di linguaggio perpetri omofobia". Se scegliessimo di usare al suo posto il verbo praticare, tradurremmo solo la parte asserita di perpetrare: dicendo pratichi l'omofobia non si esprime se la si giudichi cosa buona o cattiva. Infatti praticare è adatto ad azioni sia positive che negative: praticare la meditazione, la raccolta differenziata, l'evasione fiscale, la pesca di frodo. Volendo invece mantenere il più possibile entrambe le componenti semantiche 
principali di perpetrare, converrà scegliere il verbo commettere, perché anch'esso presuppone che si tratti di azione colpevole: commettere uninfrazione, uningiustizia, ma non commettere un atto generoso, una cortesia ecc. Come quasi sempre avviene, la sinonimia non è perfetta; perché commettere spazia su qualsiasi livello di gravità, mentre perpetrare suggerisce l'idea di una gravità molto elevata, che lo rende adatto solo per azioni molto brutte: perpetrare un crimine odioso, ma più difficilmente perpetrare una sgarberia.

Probabilmente la specializzazione di perpetrare per i valori più alti della scala di gravità si deve prima di tutto al suo essere, rispetto a commettere (che appunto ha senso più esteso), il termine meno comune. Infatti si tratta di una voce dotta, fatta nel XIV secolo reintroducendo il latino perpetrāre, che è da patrāre, 'effettuare, compiere', a sua volta da pater 'padre', cioè 'avere la paternità (di un'azione)', a cui è anteposto il prefisso per-che indica compimento, il verificarsi dell'azione in modo completo (si veda l'it. perfetto, dal lat. per + facere, 'fare completamente'). In latino il significato era quello più neutro di 'eseguire, portare a termine', senza connotazione negativa. Non si può escludere che a determinare il senso particolarmente grave dellitaliano possa avere concorso anche, per fonosimbolismo, la natura foneticamente aspra del termine, e il suo presentarsi con una reduplicazione, cioè la ripetizione della prima sillaba, che suggerisce l'idea di intensificazione, se non addirittura di accanimento. Infatti in molte lingue del mondo la reduplicazione è procedimento morfologico regolare per codificare l'intensificazione del significato. In cinese pang significa 'grasso', e pang pang 'molto grasso'. In thailandese dii è 'buono' e dii dii 'buonissimo'. In finlandese koti significa 'casa', e kotikoti 'la vera casa, quella dove si è cresciuti e non solo quella dove si vive adesso'. Del resto, che cosa significano in italiano bello bello e alto alto?

Tornando all'articolo di "Vanity Fair", è seducente l'ipotesi che chi l'ha scritto abbia voluto usare i due "strati" del significato del verbo per ottenere un effetto ricercato. Dicendo "perpetrare un delitto" non si fa niente di speciale, perché presupporre che un delitto sia male è quanto di più ovvio; ma dicendo "perpetrare un sogno" si crea un effetto di sorpresa e di sensibilizzazione dell'attenzione, con implicazioni che possono essere stilistiche e perfino letterarie. Insomma, presupponendo come se fosse ovvio e scontato che un sogno sia qualcosa di volontariamente nocivo, di criminale, di colpevole, si aprono allintelletto del lettore interpretazioni non banali della situazione descritta, e forse anche dell'esistenza in generale.

Però nell'articolo di "Vanity Fair" segnalatoci dalla lettrice si trattava di mero errore. Il contesto era questo:

Veronica Pivetti, domenica sera, si è trovata a raccontare la tragedia di Federica De Luca. Trentenne pugliese, strappata alla vita assieme a suo figlio, dal delirio egoistico di un marito padrone. Ha dovuto documentarne l'amore, la dedizione. Dare conto, attraverso una ricostruzione per immagini, di come la giovane donna abbia perpetrato il sogno di una famiglia.

La giovane, sfortunata donna di cui si parla stava solo cercando di far durare il più possibile, di perpetuare, il suo sogno su come avrebbe desiderato che fosse la sua famiglia. Di questo commovente tentativo ha approfittato lo scellerato, odioso carnefice.

Dunque, il verbo perpetrare sembra causare errori più frequentemente di altri. E non per caso l'errore di cui si tratta è lo scambio con un termine anchesso non facilissimo, e formalmente molto simile: appunto, perpetuare. Sbagli di questo genere sono ovviamente dovuti alla scarsa dimestichezza con le parole in questione, che non aiuta a riconoscere perpetuare come diverso, per forma e significato, da perpetrare. Non si tratta di un caso isolato, perché nella nostra come in altre lingue la presenza di 
termini difficili e somiglianti può indurre i parlanti a fare confusione. È quello che accade con espressioni come innestare per 'innescare', lascivo per 'permissivo, che lascia fare', leggiadro per 'leggero', quantizzare per 'quantificare', schernirsi per 'schermirsi', stentoreo per 'stentato', reticente per 'renitente'. Per alcuni di questi è troppo semplice dire che si tratti di errori, perché dall'essere comportamenti marginali si stanno diffondendo su livelli e in contesti d'uso cosi qualificati, da avviare un processo di affermazione che li rende sempre più accettabili. Ad esempio, tutti gli scambi appena citati sono ormai presenti - con diverse frequenze - nell'italiano scritto, sia del web che giornalistico. In alcuni di questi casi possiamo parlare di incipiente neosemia cioè di attribuzione a una parola di un nuovo significato che prima non aveva (il tema è introdotto da Tullio De Mauro, 2006 e ripreso, con riferimento specifico agli esempi fatti, in Lombardi Vallauri, 2018).

Per il momento gli effetti della confusione con altri termini simili restano del tutto marginali nell'uso di perpetrare, sia nel senso già visto di 'perpetuare', sia in quello che ci segnala una lettrice da Catanzaro, chiedendoci se sia corretto dire che "chi deturpa o imbratta immobili privati o pubblici è perpetrabile penalmente". Questo uso del verbo è semplicemente errato. È invece vero che chi pèrpetra un reato è poi perseguibile penalmente.

\section{Nota bibliografica:}

- De Mauro 2006 = Tullio De Mauro, Dizionarietto delle parole del futuro, Roma-Bari, Laterza, 2006.

- Fillmore $197 \mathrm{I}$ = Charles J. Fillmore, Verbs of Judging: An Exercise in Semantic Description, in Studies in Linguistic Semantics, a cura di C. J. Fillmore e D.Terence Langendoen, New York, Holt, Rinehart and Winston, I971, pp. 272-289.

- Lombardi Vallauri 2018 = Edoardo Lombardi Vallauri, Diffusione e motivazione di alcune novitá recenti nell'uso di parole italiane, in Cuadernos de Filología Italiana, 25, 2018, 79-Ioo. 